\title{
PARTIAL LEFT VENTRICULECTOMY WITH MITRAL VALVE PRESERVATION IN THE TREATMENT OF PATIENTS WITH DILATED CARDIOMYOPATHY
}

Luiz Felipe P. Moreira, MD

Noedir A. G. Stolf, MD

Edimar A. Bocchi, MD

Fernando Bacal, MD

Maria C. P. Giorgi, MD

José R. Parga, MD

Adib D. Jatene, MD
Objective: This study reports initial results of partial left ventriculectomy performed with preservation of the mitral valve in the treatment of 27 patients with idiopathic dilated cardiomyopathy. Methods: Patients were in New York Heart Association class III or IV. Partial ventriculectomy was performed as an isolated procedure in four patients and associated with mitral annuloplasty in 23 patients. There were four hospital deaths (14.8\%) and the remaining patients were followed for $11.2 \pm 6$ months. Results: Decrease of left ventricular diastolic diameter $(81.8 \pm 8.7$ to $68.5 \pm 7.6 \mathrm{~mm}, p<0.001)$ and improvement of left ventricular wall shortening $(12 \% \pm 3.1 \%$ to $18.1 \% \pm 3.9 \%, p<0.001)$ were demonstrated by echocardiography after the operation. Left ventricular radioisotopic angiography showed reduction of diastolic volume $(495 \pm 124 \mathrm{ml}$ to $352 \pm 108 \mathrm{ml}, p<0.001)$ and increase of ejection fraction $(17.7 \% \pm 4.6 \%$ to $23.7 \% \pm 8.8 \%, p<0.001)$. Right-sided heart catheterization demonstrated improvement of stroke index $\left(24.3 \pm 7.7 \mathrm{ml} / \mathrm{m}^{2}\right.$ to $28.3 \pm 7.6$ $\left.\mathrm{ml} / \mathrm{m}^{2}, p<0.01\right)$ and decrease of pulmonary wedge pressure $(23.2 \pm 8.8$ $\mathrm{mm} \mathrm{Hg}$ to $17 \pm 7 \mathrm{~mm} \mathrm{Hg}, p<0.01)$. Similar results were documented at 6 and 12 months of follow-up. Functional class improved from $3.6 \pm$ 0.5 to $1.4 \pm 0.6(p<0.001)$. However, seven patients died at midterm follow-up because of heart failure progression or arrhythmia-related events, and survival rate was $59.2 \% \pm 9.4 \%$ from 6 to 24 months of follow-up. Conclusions: Partial left ventriculectomy performed with preservation of the mitral valve improves left ventricular function and congestive heart failure in patients with dilated cardiomyopathy. Nevertheless, the high incidences of heart failure progression and arrhythmia-related deaths observed after this procedure preclude its wide clinical application. (J Thorac Cardiovasc Surg 1998;115:800-7)
Darti artial left ventriculectomy is a new surgical option introduced by Batista and coworkers ${ }^{1,2}$ for the treatment of end-stage cardiomyopathies. Its primary objective is the reduction of left ventricular (LV) wall tension by the decrease of chamber

From the Instituto do Coração da Faculdade de Medicina da Universidade de São Paulo, São Paulo, Brazil.

Supported by grant 1058/95/58 from Fundação E. J. Zerbini, São Paulo, Brazil.

Received for publication July 10, 1997; revisions requested Oct. 10, 1997; revisions received Nov. 11, 1997; accepted for publication Nov. 11, 1997.

Address for reprints: Luiz Felipe P. Moreira, MD, Divisão de Cirurgia-Instituto do Coração, Av. Dr. Enéas Carvalho Aguiar, 44, São Paulo, SP, Brazil. CEP:05403-000

Copyright (C) 1998 by Mosby, Inc.

$0022-5223 / 98 \$ 5.00+0 \quad \mathbf{1 2 / 1 / 8 7 5 2 7}$ volume/mass relationship, which is obtained by the resection of a large portion of $\mathrm{LV}$ free wall.

No experimental data exist regarding the benefits of $\mathrm{LV}$ chamber reduction in heart failure models. In addition, despite the existence of initial clinical reports of LV function early improvement after partial ventriculectomy, ${ }^{3-7}$ no documentation is found regarding long-term survival or prolonged benefits after this procedure in patients with dilated cardiomyopathy.

Accordingly, a prospective study concerning the effects of partial left ventriculectomy in the treatment of severe cardiomyopathies has been conducted by the Heart Failure and Heart Transplantation Program of the São Paulo Heart Institute since 1995. This report shows the initial results of this procedure, performed with preservation of the mitral valve, in the treatment of advanced heart failure caused by idiopathic dilated cardiomyopathy. 


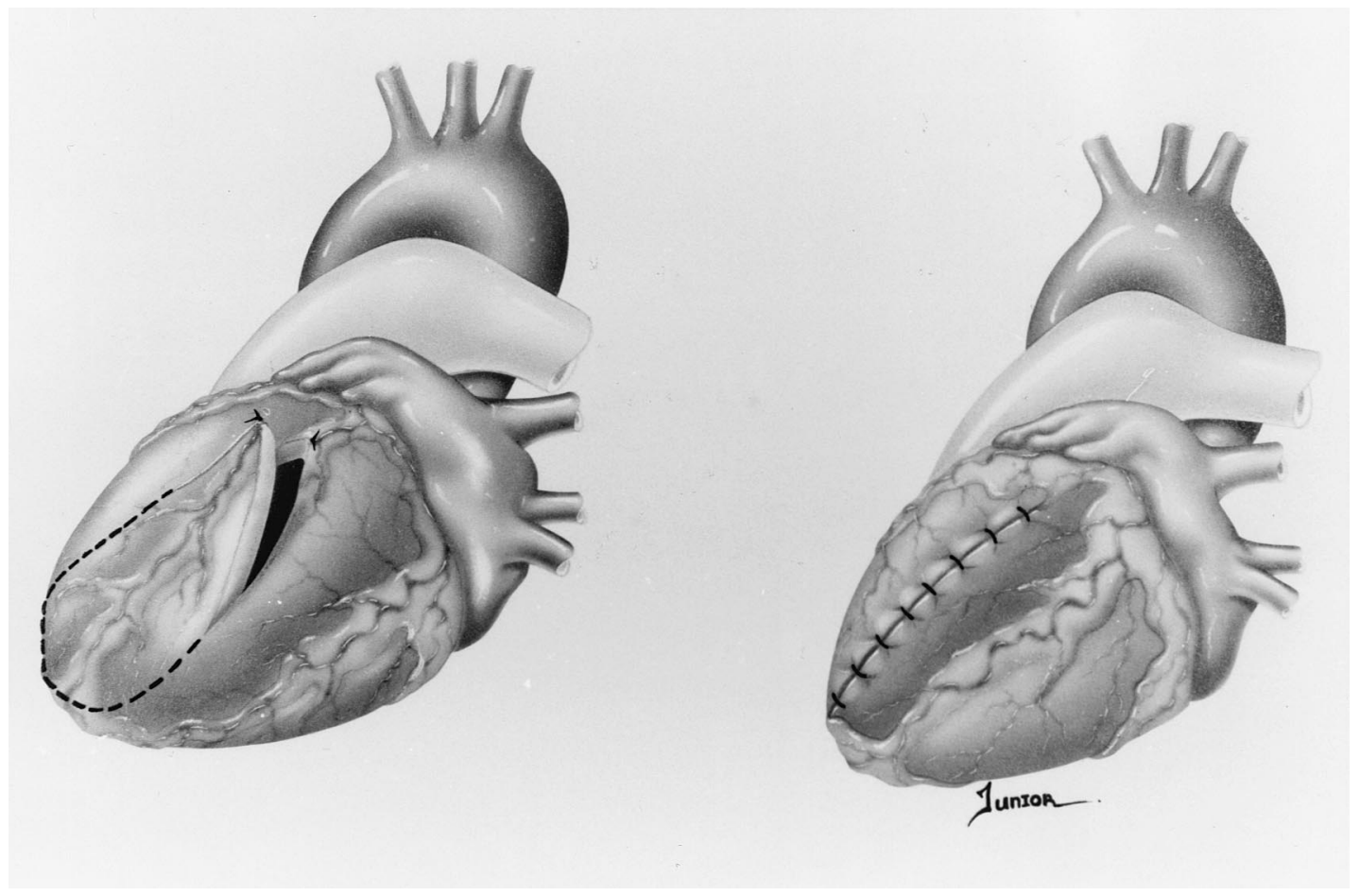

Fig. 1. Schematic representation of partial left ventriculectomy.

\section{Methods}

Patient population. Entry into the protocol was based on the existence of significant functional limitation despite attempts to optimize medical therapy with maximal doses of diuretics and angiotensin converting enzyme inhibitors or vasodilators. Patients also had reduced LV function characterized by radioisotopic ejection fraction $\leq 25 \%$ and persistently high filling pressures. Medical or psychosocial contraindications to heart transplantation were present or this procedure was refused by the patients. They signed a special informed consent form according to the Ethical and Scientific Review Board of the Heart Institute.

From April 1995 to December 1996, partial ventriculectomy was performed in 27 patients with idiopathic dilated cardiomyopathy. The patients' ages ranged from 33 to 72 years (mean 47.3 years); and 23 patients were men. Eleven patients were in New York Heart Association (NYHA) class III and 16 were in persistent class IV. The mean duration of symptoms was 4.2 years, and they had an average of 2.4 hospitalizations for heart failure treatment in the preceding year. Five of these patients also had reversible cardiogenic shock with temporary use of intravenous inotropic drugs.

Preoperative LV ejection fractions ranged from $9 \%$ to $25 \%$ (mean $17.7 \%$ ), as documented by radioisotopic scintigraphy. Patients also had a mean LV end-diastolic pressure of $24.3 \pm 9.2 \mathrm{~mm} \mathrm{Hg}$ and a mean cardiac index of $2.08 \pm 0.49 \mathrm{~L} / \mathrm{min} \cdot \mathrm{m}^{2}$ at heart catheterization. Absence of significant coronary artery compromise was also shown in every patient. Mitral valve insufficiency was present in 23 patients, being moderate in 5 patients and mild in 18 patients. Five patients also had significant tricuspid valve insufficiency, being moderate in four patients and severe in one patient.

Twenty-five patients were in sinus rhythm and the other two were in atrial fibrillation. Nonsustained ventricular tachycardia episodes were documented by Holter recordings in 16 patients, whereas the occurrence of sustained ventricular tachycardia not controlled by medical therapy was considered a contraindication for this operation.

Surgical procedure. Partial left ventriculectomy was performed as an isolated procedure or associated with atrioventricular valve annuloplasty. A median sternotomy approach was used with a standard cardiopulmonary bypass. All procedures were usually done under normothermia and on the beating heart. De-airing was accomplished through the opened left chambers supplemented by an aortic root suction catheter.

LV volume reduction was performed according to the technique initially described by Batista et al. ${ }^{1}$ A slice of the ventricular myocardium as large as possible was resected between the papillary muscles from the apex of the heart up to $2 \mathrm{~cm}$ from the mitral anulus as represented in Fig. 1. Obtuse marginal branches of the circumflex artery were usually resected with the myocardial specimen. The left ventricle was then repaired with a double 3-0 polypropylene suture anchored in bovine pericardium strips.

Mitral valve annuloplasty was done in the presence of any degree of mitral regurgitation to avoid the influence of residual insufficiency and to contribute to LV dimen- 


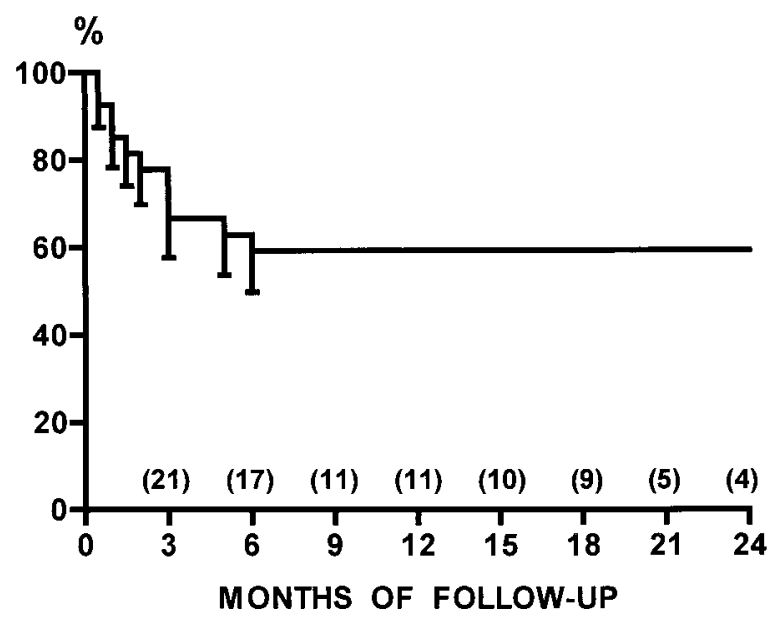

Fig. 2. Actuarial survival curve after partial left ventriculectomy. Numbers in parentheses indicate patients at risk in the follow-up period. Values are presented as mean \pm standard error of the mean.

sion decrease. The mitral valve was assessed through the left atrium, and the mitral anulus was reduced by plication of the posterior leaflet region with sutures anchored in a strip of bovine pericardium. The De Vega tricuspid annuloplasty was done in patients with moderate to severe tricuspid regurgitation. Endocardial automatic cardioverter-defibrillators were routinely implanted in the last eight patients after discharge from the intensive care unit.

Follow-up protocol. Patients were clinically evaluated on a monthly basis during the first 3 months of follow-up and at least every 6 months afterward. Patients continue to use diuretics and angiotensin-converting enzyme inhibitors or vasodilators with approximately the same doses as before the operation. Amiodarone was routinely used in patients who had episodes of sustained ventricular tachycardia or to decrease heart rate in patients with atrial fibrillation in doses ranging from 200 to $400 \mathrm{mg} /$ day. Ventricular function was analyzed before the operation, during the first postoperative month, and at 6 and 12 months of follow-up by means of echocardiography, radioisotopic scintigraphy, magnet resonance imaging, and right-sided heart catheterization.

Doppler echocardiography was performed with the Aloka SSD-730 and SSD-870 sector scans (Aloka Inc., Tokyo, Japan). LV end-diastolic and end-systolic diameters were obtained by M-mode and two-dimensional echocardiograms. Percentage of fractional shortening was calculated using standard formulas. The severity of mitral and tricuspid valve insufficiency was determined by Doppler analysis.

Radioisotopic scintigraphy was obtained after in vivo labeling of red blood cells by ${ }^{99 m}$ Tc. Gated blood pool imaging was acquired in the left anterior oblique view with a Siemens model LEM+ camera (Siemens Corp., Union, N.J.). The images were analyzed in a Microvax model 3300 computer (Siemens): LV volumes and ejection frac- tion were calculated without the use of correcting factors to avoid mitral regurgitation influence.

Noninvasive evaluation of LV function was also done by magnetic resonance imaging. This study was performed using a $0.5 \mathrm{~T}$ magnet (Phillips, Best, The Netherlands), with cine magnet resonance imaging sequences to measure volumes and to calculate ejection fraction.

For right-sided heart catheterization, a thermodilution balloon-tipped catheter was positioned in the left pulmonary artery. Cardiac output was obtained by the thermodilution technique, whereas arterial pressures were measured by noninvasive standard means. Cardiac index, stroke index, left and right ventricular stroke work indexes, and vascular resistances were calculated by standard formulas.

Statistical analysis. Data were analyzed using the Statistics Data Analysis Package (Stata Corporation, College Station, Tex.). Cumulative mortality and event rates over time were determined by the Kaplan-Meier method, and differences between them were assessed by the log-rank test. Repeated measures analysis of variance and Dunnet's test were used to compare data obtained before and after partial left ventriculectomy. Data are presented as mean \pm standard deviation, whereas survival rates are presented as mean \pm standard error of the mean. Linear regression was used to test the relationship between some variables.

\section{Results}

Immediate surgical results. Isolated partial left ventriculectomy was performed only in four patients. This procedure was associated with mitral valve annuloplasty in the remaining 23 patients and 5 of them also underwent the De Vega tricuspid valve annuloplasty. The resected myocardial specimen measured $10.7 \pm 1.3 \mathrm{~cm}$ in length and $4.8 \pm 0.8$ $\mathrm{cm}$ in width. All patients were weaned from cardiopulmonary bypass with dobutamine and sodium nitroprusside, and eight patients also required intraaortic balloon counterpulsation. Intraoperative transesophageal echocardiography after discontinuation of bypass revealed no mitral regurgitation in 22 patients and mild regurgitation in 5 patients.

Pharmacologic inotropic support was usually maintained for 1 week after the operation, and intraaortic balloon pumping was normally discontinued the second or third postoperative day. One patient had refractory heart failure and required the insertion of a centrifugal pump for LV assistance the second day. Another patient had dehiscence of the mitral valve annuloplasty and was reoperated for mitral valve replacement 5 days after the initial operation.

Six patients $(22.2 \%)$ had episodes of sustained ventricular tachycardia reverted by electrical cardioversion during the first 2 weeks of follow-up. Tran- 
Table I. Noninvasive evaluation of $L V$ function

\begin{tabular}{|c|c|c|c|c|c|}
\hline & \multirow[b]{2}{*}{ Preoperative } & \multicolumn{3}{|c|}{ Follow-up } & \\
\hline & & $1 \mathrm{mo}$ & $6 \mathrm{mo}$ & $12 \mathrm{mo}$ & \\
\hline Echocardiography & $(n=23)$ & $(n=23)$ & $(n=16)$ & $(n=10)$ & \\
\hline $\operatorname{LVEDD}(\mathrm{mm})$ & $81.8 \pm 8.7$ & $68.5 \pm 7.6^{*}$ & $70.2 \pm 7 *$ & $71.1 \pm 6.6^{*}$ & $p<0.001$ \\
\hline LVSWS (\%) & $12 \pm 3.1$ & $18.1 \pm 3.9^{*}$ & $16.4 \pm 3.5^{*}$ & $16.5 \pm 5.1^{*}$ & $p=0.008$ \\
\hline $\begin{array}{r}\text { Radioisotopic } \\
\text { scintigraphy }\end{array}$ & $(n=23)$ & $(n=23)$ & $(n=16)$ & $(n=10)$ & \\
\hline $\operatorname{LVEDV}(\mathrm{ml})$ & $495 \pm 124$ & $352 \pm 108^{*}$ & $384 \pm 95^{*}$ & $380 \pm 70^{*}$ & $p=0.001$ \\
\hline LVEF $(\%)$ & $17.7 \pm 4.6$ & $23.7 \pm 8.8^{*}$ & $24.1 \pm 8.3^{*}$ & $23.7 \pm 6$ & $p=0.001$ \\
\hline $\begin{array}{l}\text { Magnetic resonance } \\
\text { imaging }\end{array}$ & $(n=20)$ & $(n=20)$ & $(n=7)$ & & \\
\hline LVEDV (ml) & $371 \pm 118$ & $272 \pm 89^{*}$ & $286 \pm 90 *$ & - & $p<0.001$ \\
\hline LVEF (\%) & $16.7 \pm 7.5$ & $29.1 \pm 9.1^{*}$ & $24.6 \pm 7.2^{*}$ & - & $p=0.01$ \\
\hline
\end{tabular}

$L V E D D$, Left ventricular end-diastolic diameter; $L V S W S$, left ventricular segmental wall shortening; $L V E D V$, left ventricular end-diastolic volume; $L V E F$, left ventricular ejection fraction. Numbers in parentheses indicate the patients evaluated at each time. Value of $p$ obtained by analysis of variance in patients with complete follow-up.

${ }^{*} p<0.05$ in relation to preoperative data of matched patients.

sient periods of renal failure developed in four patients $(14.8 \%)$, and pulmonary complications occurred in five patients (18.5\%). The mean peak serum level of the myocardial component of the creatine kinase enzyme was $116 \pm 58$ IU.

Four patients died during the hospitalization period $(14.8 \%)$. The causes of death were cardiogenic shock associated with restrictive LV dysfunction in the patient who required mechanical circulatory support with centrifugal pump; septicemia and cardiogenic shock in the patient reoperated because of mitral annuloplasty dehiscence; incessant sustained ventricular tachycardia in one patient; and bleeding associated with disseminated intravascular coagulation in another patient.

Midterm follow-up. Twenty-three patients were discharged from the hospital, and they were followed from 2 to 26 months (mean 11.2 months), corresponding to 265 patient-months. At hospital discharge, NYHA class improved from $3.6 \pm 0.5$ to $1.4 \pm 0.6(p=0.001)$.

During the first 6 months of follow-up, five patients were rehospitalized because of heart failure progression and the other two because of sustained ventricular tachycardia episodes. Four of these patients were listed for heart transplantation, and this procedure was urgently done in one of them 7 months after partial left ventriculectomy.

Furthermore, seven patients died at midterm follow-up. The mortality risk was $4.1 \%$ per patientmonth, and the survival rates were $66.6 \% \pm 9.1 \%$ at 3 months and $59.2 \% \pm 9.4 \%$ from 6 to 24 months of follow-up (Fig. 2). Cause of death was progressive heart failure in three patients, which was associated with pulmonary thromboembolism in one of them. The other four patients died from incessant sustained ventricular tachycardia or suddenly. The incidences of heart failure progression leading to death or the necessity for urgent heart transplantation and of arrhythmia-related deaths were $2.2 \%$ and $1.9 \%$ per patient-month, respectively. Two of the patients who had an arrhythmia-related death were awaiting heart transplantation because of heart failure decompensation. Three of them had previous episodes of sustained ventricular tachycardia; two were taking amiodarone, and one had an automatic internal cardioverter-defibrillator. Besides one sudden death, another of the eight patients who received cardioverter-defibrillators died from heart failure progression, representing a risk of mortality of $3.7 \%$ per patient-month.

On the other hand, the 16 patients who survived more than 6 months of follow-up maintained significant clinical improvement. Ten of these patients are currently in functional class I and six are in class II $(p<0.001$ in relation to preoperative data). Furthermore, only one of them required hospitalization twice because of heart failure symptoms.

Ventricular function evaluation. Data obtained by echocardiography, radioisotopic scintigraphy, and magnetic resonance imaging are shown in Table I. Significant improvements of LV fractional shortening and ejection fraction were documented by the three methods early after partial ventriculectomy. These findings occurred in the presence of the decrease of LV end-diastolic diameter and volume in the order of $30 \%$. At 6 and 12 months of follow-up, the results of echocardiography and ra- 
Table II. Hemodynamic evaluation

\begin{tabular}{|c|c|c|c|c|c|}
\hline & \multirow[b]{2}{*}{$\begin{array}{l}\text { Preoperative } \\
\quad(n=23)\end{array}$} & \multicolumn{3}{|c|}{ Follow-up } & \\
\hline & & $\begin{array}{c}1 \text { mo } \\
(n=23)\end{array}$ & $\begin{array}{c}6 \text { mo } \\
(n=16)\end{array}$ & $\begin{array}{c}12 \text { mo } \\
(n=10)\end{array}$ & \\
\hline MRAP (mm Hg) & $10 \pm 5.5$ & $7.8 \pm 4.9$ & $7.5 \pm 5.4$ & $6.7 \pm 5.1$ & $p=0.12$ \\
\hline MPAP (mm Hg) & $32.2 \pm 9.5$ & $29 \pm 9.3$ & $29.3 \pm 14.1$ & $24 \pm 13.6$ & $p=0.13$ \\
\hline MPWP (mm Hg) & $23.2 \pm 8.8$ & $17 \pm 7^{*}$ & $19.5 \pm 9.8$ & $20.4 \pm 8.6$ & $p=0.03$ \\
\hline $\mathrm{MAP}(\mathrm{mm} \mathrm{Hg})$ & $78 \pm 15.3$ & $80.3 \pm 17.6$ & $88 \pm 20.4^{*}$ & $83.4 \pm 22^{*}$ & $p<0.001$ \\
\hline $\mathrm{CI}\left(\mathrm{L} / \mathrm{min} \cdot \mathrm{m}^{2}\right)$ & $2.13 \pm 0.54$ & $2.5 \pm 0.58^{*}$ & $2.63 \pm 0.4^{*}$ & $2.6 \pm 0.5^{*}$ & $p=0.007$ \\
\hline $\mathrm{SI}\left(\mathrm{ml} / \mathrm{m}^{2}\right)$ & $24.3 \pm 7.7$ & $28.3 \pm 7.7^{*}$ & $33.1 \pm 6.4^{*}$ & $33 \pm 6.9^{*}$ & $p=0.006$ \\
\hline LVSWI $\left(\mathrm{gm} \cdot \mathrm{m} / \mathrm{m}^{2}\right)$ & $19.7 \pm 9.1$ & $26.1 \pm 9.9^{*}$ & $33 \pm 10.5^{*}$ & $31.1 \pm 7.9^{*}$ & $p<0.001$ \\
\hline PVR (dyne $\cdot \mathrm{sec} \cdot \mathrm{cm}^{-5}$ ) & $197 \pm 144$ & $228 \pm 152$ & $177 \pm 101$ & $127 \pm 52$ & $p=0.33$ \\
\hline
\end{tabular}

$M R A P$, Mean right atrial pressure; $M P A P$, mean pulmonary artery pressure; $M P W P$, mean pulmonary wedge pressure; $M A P$, mean arterial pressure; $C I$, cardiac index; SI, stroke index, $L V S W I$, left ventricular stroke work index; $P V R$, pulmonary vascular resistance. Numbers in parentheses indicate the patients evaluated at each time. Value of $p$ obtained by analysis of variance in patients with complete follow-up.

${ }^{*} p<0.05$ in relation to preoperative data of matched patients.

dioisotopic scintigraphy were approximately the same. Magnetic resonance imaging also showed similar data at the 6-month follow-up. Besides these changes, Doppler echocardiography showed that the degree of mitral and tricuspid valve insufficiency did not change in the follow-up period.

Table II displays the data obtained in the hemodynamic studies. Significant improvements of the cardiac index, stroke index, and LV stroke work index were found early after the operation, associated with a significant decrease of mean pulmonary wedge pressure. Similar results were observed in the surviving patients at 6 and 12 months of follow-up.

The modifications of LV function after partial ventriculectomy were comparable only in patients who underwent this procedure and when it was associated with mitral valve annuloplasty. No correlation exists between radioisotopic LV ejection fraction changes and the percentage of the myocardium that was resected or LV end-diastolic volume modifications as exemplified in Fig. 3, $A$ and $B$. The percentage of myocardial resection was based on the echocardiographic estimation of LV perimeter.

On the other hand, significant improvement of radioisotopic LV ejection fraction occurred early after the operation only in patients who survived long term $(18.3 \% \pm 5.1 \%$ to $26.5 \% \pm 8.3 \%, p<$ $0.001)$. This parameter did not change in patients who died or who had urgent heart transplantation $(17.8 \% \pm 4.7 \%$ to $17.1 \% \pm 5.3 \%)$. The same trend was not observed by echocardiographic and magnetic resonance studies, and the modifications of hemodynamic parameters were also more significant in patients surviving long term.

\section{Discussion}

The key event leading to heart failure is the loss of a critical quantity of functioning myocardial cells. Compensatory mechanisms include the activation of hemodynamic and neurohormonal modifications to enhance the contractile force of the noninjured myocardium, preserving cardiac function. ${ }^{8,9}$ Nevertheless, these mechanisms introduce important risk factors. Chamber dilatation increases the internal ventricular wall stress, leading to accelerated energy expenditure. ${ }^{9,10}$ Furthermore, potential development of mitral valve insufficiency may overload the already dilated left ventricle, compromising the compensatory remodeling process. ${ }^{11}$ Limiting or reversing chamber dilatation is an important therapeutic goal in standard medical therapy, the results of which only show modest effects on LV remodeling, however. ${ }^{8}$ On the other hand, these concepts also provide the rationale for operative techniques to reverse the chamber remodeling process.

Ventricular function changes. This study shows that partial left ventriculectomy, associated when necessary with mitral valve annuloplasty, significantly improves LV function. The increase of LV ejection fraction was confirmed by different methods and occurred combined with significant decreases in end-diastolic diameters and volume. Significant modifications of hemodynamic parameters were also found. More precisely, improvements in cardiac index, stroke index, and LV stroke work index were shown in the presence of decreased pulmonary wedge pressure. Besides these changes, the enhancement of LV function after partial ventriculectomy involves modifications in the mechanical prop- 


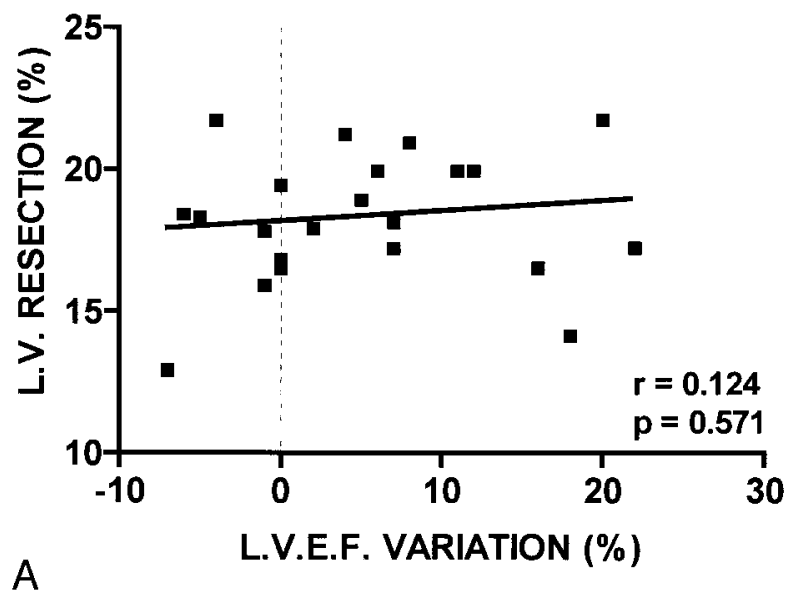

A

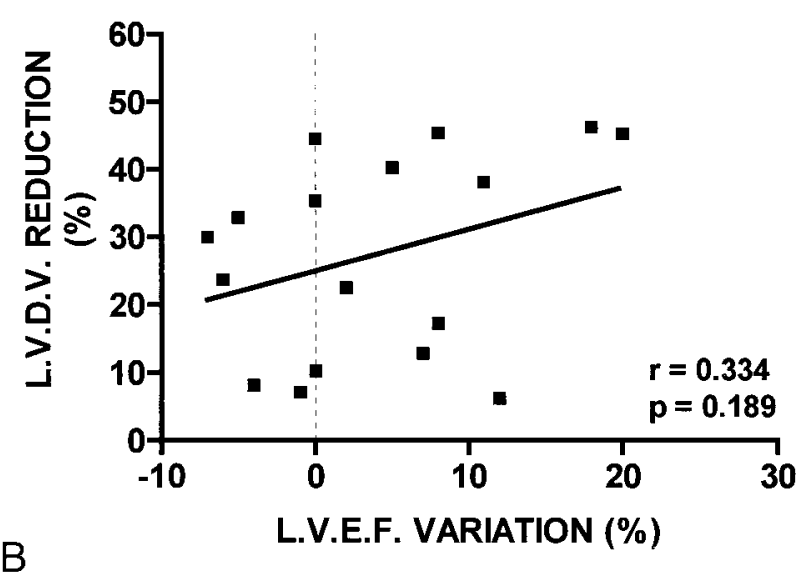

Fig. 3. A, Plots of radioisotopic LV ejection fraction (L.V.E.F.) variations after partial left ventriculectomy in relation to the percentage of left ventricular (L.V.) myocardial resection. B, Plots of radioisotopic left LV ejection fraction (L.V.E.F.) variations after partial left ventriculectomy in relation to LV diastolic volume (L.V.D.V.) reduction.

erties of the failing heart, as observed in another study performed in 11 patients of the current population. ${ }^{3}$ Significant reductions of LV end-systolic and end-diastolic stresses were demonstrated at the first month of follow-up by the determination of pressure-volume loops. Moreover, that study documented the increase of maximal elastance and decreases in end-diastolic pressure and in muscle and chamber stiffness after the operation.

Improvement of the contractile characteristics of the failing myocardium has been also observed after other operative interventions. Dynamic cardiomyoplasty is responsible for modifications in hemodynamic and mechanical parameters of LV function comparable to those documented in this investigation. ${ }^{12-14}$ Another method that can result in improved myocardial contractility and reversion of chamber dilatation is the chronic use of mechanical assist devices. The continuous ventricular chamber unloading obtained with this procedure may lead to a marked reduction in myocytolysis and to an enhanced pressure-volume relationship at the time of the device explantation. ${ }^{15,16}$

Maintenance of LV function improvement for up to 12 months after partial ventriculectomy in this series appears to indicate that heart failure stops progressing in the surviving patients, as similarly documented with dynamic cardiomyoplasty. ${ }^{14} \mathrm{Nev}-$ ertheless, longer term follow-up will be necessary to confirm the existence of prolonged benefits.

Influence of technical aspects. Several issues exist regarding partial left ventriculectomy technical aspects. Nevertheless, this series shows that a significant reduction of LV cavity size was obtained in patients with dilated cardiomyopathy, performing the myocardium resection without papillary muscle resection or translocation. Although other authors advocate a more aggressive approach regarding the myocardial resection, with the necessity of associated mitral valve replacement or papillary muscles translocation in more than $30 \%$ of their cases, similar results have been described in those series in relation to ventricular function modifications. ${ }^{2,5,6}$ Furthermore, this study showed the absence of correlation between LV ejection fraction changes and the degree of myocardium resection or the level of LV end-diastolic volume reduction.

The impact of mitral valve reconstruction and its association with partial left ventriculectomy remains uncertain. The isolated correction of severe mitral regurgitation by means of remodeling annuloplasty is responsible for significant modifications in LV function. Improvement of LV ejection fraction was documented after that procedure by echocardiography in 16 patients with dilated cardiomyopathy in the presence of slight modification of LV enddiastolic volume. ${ }^{17}$ This finding was also associated with a favorable clinical outcome with a 1-year actuarial survival of $75 \%$. On the other hand, although the changes in ejection fraction in the current study were similar to those obtained with isolated mitral valve reconstruction, most of our patients had only mild or moderate mitral regurgitation before the operation, justifying a less substan- 
tial impact of mitral annuloplasty on LV function. Moreover, three patients who only had partial left ventriculectomy in this series also showed important ejection fraction improvement (from $18.1 \% \pm 3.9 \%$ to $25.2 \% \pm 11.2 \%$ ). Accordingly, the mechanisms of action of these procedures may be complementary and their association with the treatment of patients with mitral regurgitation caused by dilated cardiomyopathies is justified.

Functional improvement and survival. The improvement of LV function after partial ventriculectomy seems to be responsible for the amelioration of patients' functional class, which agrees with the general clinical experience with this procedure. ${ }^{2,4,5}$ Most of our surviving patients were in NYHA class I or II for more than a year of follow-up, and the number of hospitalizations per patient per year also decreased.

Nevertheless, clinical improvement occurred only in a percentage of patients undergoing partial left ventriculectomy. Immediate and early mortalities after this procedure were high and occurred because of heart failure progression or arrhythmia-related events. The same situation was observed in Batista's experience, ${ }^{2}$ although the existence of different causes and other associated procedures makes comparison less accurate.

Absence of significant LV function improvement after partial left ventriculectomy (radioisotopic ejection fraction increases of less than 10\%) occurred in $33 \%$ of the patients in this series and was associated with increased mortality or necessity of urgent heart transplantation during the first 6 months of followup. Similar findings were observed by Starling and colleagues that showed the necessity of heart transplantation or mechanical circulatory support in more than $20 \%$ of their patients in the first postoperative months. ${ }^{5,6}$ Also important is the fact that either patients who died because of heart failure progression or an arrhythmia-related event had lack of $\mathrm{LV}$ function improvement immediately after the operation in this series.

On the other hand, the elevated incidence of ventricular arrhythmias after partial left ventriculectomy seems to involve mechanisms related to the procedure itself. The existence of areas of fibrosis and myocardial infarction around the ventricular scar may modify the electrophysiologic substrate leading to ventricular arrhythmias in dilated cardiomyopathy patients, ${ }^{18}$ perhaps by new reentrant pathways. The early introduction of antiarrhythmic drugs or the implantation of automatic cardioverter- defibrillators could decrease this complication or its consequences. The reduction of the incidence of sudden cardiac death in patients waiting for heart transplantation is described by some authors with these procedures. ${ }^{19,20}$ However, three of the five patients who died suddenly or as a result of reentrant sustained ventricular tachycardia in this series were taking amiodarone or had an automatic cardioverter-defibrillator.

The important early incidence of heart failure progression and arrhythmia-related deaths observed by several authors after partial left ventriculectomy clearly indicates the necessity of identifying the determinants of unfavorable outcome. However, the cohort of patients in this study is too small to perform any formal prognostic factor analysis, and there is not any other report demonstrating factors associated with the adverse results of this procedure. Otherwise, the absence of significant modifications in radioisotopic LV ejection fraction and hemodynamic parameters observed in patients who did not survive long term in this series is an important finding in identifying patients who need heart transplantation after partial left ventriculectomy.

A possible reason to have an absence of improved myocardial contractility in response to $\mathrm{LV}$ volume reduction in patients with severe cardiomyopathies may be related to the condition of the myocardial cells. Compensatory mechanisms of heart failure can cause irreversible structural remodeling of the heart because of slippage and elongation of myocardial fibers. ${ }^{9}$ These findings are, however, difficult to identify by standard methods. Otherwise, evaluation of myocardium specimens obtained by endomyocardial biopsy by use of refined histologic and histochemical examinations may potentially contribute to separate out these failures.

Study limitations. This study has several limitations, but it is the first to analyze the possible impact of partial left ventriculectomy in the treatment of severe heart failure caused by idiopathic dilated cardiomyopathy. Although the association of this procedure with mitral valve annuloplasty in most of our patients precluded definitive conclusions about its value, the significant improvement of LV function documented for up to 12 months of follow-up is remarkable and very encouraging.

The absence of a matched group of patients managed medically impeded any judgment about the safety and benefits of partial left ventriculectomy. The elevated mortality observed in this study, however, indicates that institutions investigating this 
procedure should provide postoperative access to alternate options of treatment, including heart transplantation and mechanical circulatory support, for all patients. A more aggressive use of mechanical ventricular assist devices as bridges to heart transplantation could yield a decrease in mortality in the immediate and early postoperative periods as demonstrated by Starling and coworkers. 5, 6

\section{Conclusions}

Despite many controversies about this operative procedure, this investigation demonstrated that partial left ventriculectomy, performed with preservation of the mitral valve, improves LV function in patients with idiopathic dilated cardiomyopathy. Although this procedure seems to ameliorate heart failure symptoms in the surviving patients, the high incidence of heart failure progression and arrhythmia-related deaths in the early postoperative period precludes its wide clinical application. However, further studies need to be conducted to improve technical aspects, immediate postoperative management, and proper patient selection for partial left ventriculectomy. The effective management of postoperative ventricular arrhythmias and the long-term outcome of patients who survive the early postoperative period are also points that need to be addressed by future investigations.

\section{REFERENCES}

1. Batista RJV, Santos JLV, Takeshita N, Bocchino L, Lima PN, Cunha MA. Partial left ventriculectomy to improve left ventricular function in end-stage heart disease. J Card Surg 1996;11:96-7.

2. Batista RJV, Santos JLV, Nery P, et al. Partial left ventriculectomy to treat end-stage heart disease. Ann Thorac Surg 1997;64:634-8.

3. Bellotti G, Moraes A, Bocchi EA, et al. Effects of partial ventriculectomy on left ventricular mechanical properties, shape and geometry in patients with dilated cardiomyopathy. Arq Bras Cardiol 1996;67:395-400.

4. Bombonato R, Bestetti RB, Sgarbieri R, et al. Initial experience with partial left ventriculectomy in the treatment of terminal cardiac insufficiency. Arq Bras Cardiol 1996;66:189-92.
5. McCarthy PM, Starling RC, Wong J, Scalia GM, Buda T, Vargo RL, et al. Early results with partial left ventriculectomy. J Thorac Cardiovasc Surg 1997;114:755-65.

6. Starling RC, Young JB, Scalia GM, et al. Preliminary observations with ventricular remodeling surgery for refractory congestive heart failure. J Am Coll Cardiol 1997;29:64A.

7. Takeshita N, Kawaguchi AT, Lima PRN, et al. Hemodynamic changes in patients undergoing left ventricular diameter reduction (Batista operation). J Am Coll Cardiol 1997;29:64A.

8. Cohn JN. Structural basis for heart failure: ventricular remodeling and its pharmacological inhibition. Circulation 1995;91:2504-7.

9. Packer M. Pathophysiology of chronic heart failure. Lancet 1992;340:88-92.

10. Lee TH, Hamilton MA, Stevenson LW, et al. Impact of left ventricular cavity size on survival in advanced heart failure. Am J Cardiol 1993;72:672-6.

11. Blondheim DS, Jacobs LE, Kotler MN, Costacurta GA, Parry WR. Dilated cardiomyopathy with mitral regurgitation: decreased survival despite a low frequency of left ventricular thrombus. Am Heart J 1991;122:763-71.

12. Bellotti G, Moraes A, Bocchi EA, et al. Late effects of cardiomyoplasty on left ventricular mechanics and diastolic filling. Circulation 1993;88:II-304-8.

13. Moreira LFP, Bocchi EA, Stolf NAG, Pileggi F, Jatene AD. Current expectations in dynamic cardiomyoplasty. Ann Thorac Surg 1993;55:299-303.

14. Moreira LFP, Stolf NAG, Bocchi EA, et al. Clinical and left ventricular function outcomes up to five years after dynamic cardiomyoplasty. J Thorac Cardiovasc Surg 1995;109:353-62.

15. Frazier OH, Benedict CR, Radovancevic B, et al. Improved left ventricular function after chronic left ventricular unloading. Ann Thorac Surg 1996;62:675-82.

16. Levin HR, Oz JM, Packer M, Rose E, Burkhoff D. Reversal of chronic ventricular dilatation in patients with end-stage cardiomyopathy by prolonged mechanical unloading. Circulation 1995;91:2717-20.

17. Bolling SF, Deeb GM, Brunsting LA, Bach DS. Early outcome of mitral valve reconstruction in patients with end-stage cardiomyopathy. J Thorac Cardiovasc Surg 1995;109:676-83.

18. Stevenson WG. Mechanisms and management of arrhythmias in heart failure. Cur Opin Cardiol 1995;10:274-81.

19. Doval HC, Nul DR, Grancelli HO, Perrone SV, Bortman GR, Curiel R. Randomised trial of low-dose amiodarone in severe congestive heart failure. Lancet 1994;344:493-8.

20. Sweeney MO, Ruskin JN, Garan H, et al. Influence of the implantable cardioverter/defibrillator on sudden death and total mortality in patients evaluated for cardiac transplantation. Circulation 1995;92:3273-81. 